\title{
Human Audio/Vestibular System: Data Input Channels for Robotic Force and Moment Sensor Measurements
}

\author{
Sherry Draisey \\ Good Vibrations Engineering Ltd \\ sherry@gve.on.ca
}

\author{
Mayes Mullins \\ Good Vibrations Engineering Ltd. \\ crs0546@ca.inter.net
}

\begin{abstract}
The design goal was development of an intuitive human machine interface for force and moment data from space robotic operations. This paper defines overall requirements and goals. It describes experimental approaches used to evaluate our 'nature' inspired solution. The final portion of the paper discusses the design and prototyping of the segment of the problem which has lead to our first product.

One of nature's ways of presenting multiple degree of freedom (dof), vector data is through our audio and vestibular systems. This directional capability is being applied as a human machine interface (HMI) for robotic force sensing.

Human audio direction ability is accurate except for sounds generated above and behind our heads. This inaccuracy has lead us to the development of the vestibulator.

The vestibulator is a wireless device which applies low levels of current, to the human subject mastoid bones through surface mounted electrodes. These induce perceptions of tilt. The polarity of the signals provide directional stimulus.
\end{abstract}

\section{Introduction}

From a design perspective, the work described in this paper follows the milestones of:

$\begin{array}{ll}\text { - } & \text { goal } \\ \text { - } & \text { design concept/inspiration } \\ \text { - } & \text { requirements } \\ \text { - } & \text { design approach } \\ \text { - } & \text { design } \\ & \text { prototype build }\end{array}$

It started with a goal to add more human capabilities, i.e. force sensing, to telerobotic operations. It has split into two streams. Figure 1, shows the design path which has lead from our original goal to the vestibulator prototypes.

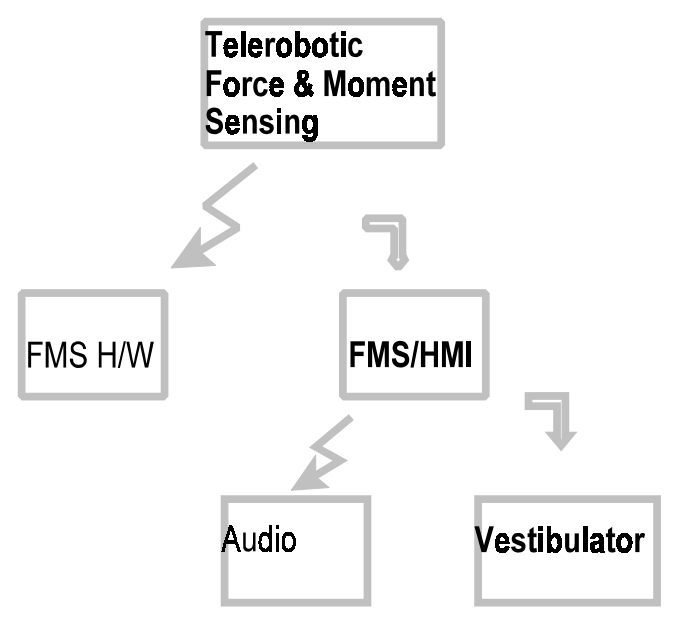

Figure 1

Engineering Problem Decomposition

Teleoperational robotic manoeuvres are not as common in the robotic world as 'pick and place' robotics. This may account for the lack of force sensors implemented in robotic applications. The Canadarms (Shuttle Remote Manipulator Systems, SRMS) have no force sensor, though there was an experimental one flown in 1994 (STS-62). The Canadarm2 and SPDM (Special Purpose Dextrous Manipulator) have both been built with force sensors, but they have yet to be operationally utilized. There 
are certainly tasks one can imagine that require force sensing at the tip - such as washing a window, but these tasks are not yet required. The human-robotic force feedback problem remains significant.

While we continue in our efforts to improve force moment sensor capabilities, this paper deals with the HMI (human machine interface) problem. A simple software demonstration of the audio aspect of the HMI approach is presented. The actual design and prototype build work described is for the vestibulator hardware/software portion of such an HMI system. The vestibulator is a wireless electronic device for use on human subjects.

\section{Teleoperational Scenario}

The requirements which we have based our design on have been derived from the Canadian space robots.

The original force moment sensor design considerations began in the early 80 's and were based on the idea of upgrading the shuttle Canadarm to include force sensing. The HMI problem which is addressed in this paper has been targeted towards the Canadarm2 and began its development in 1996 [1]. Two systems of presenting directional data were the basis of 'inspiration' for the design to meet the HMI requirement - audio and vestibular.

The need for robotic force sensing seems intuitively obvious, when we consider the ultimate robot - the human. Humans have many types of force and pressure sensors (tactile, proprioceptive) distributed throughout our 'arm' system. The difference between human force sensing and teleoperational force and moment sensing for a space environment is twofold. A space sensor requires a wide dynamic range and must be very stiff, due to it position near the tip of a long arm (15.3 metre shuttle arm, and 17 metre station arm). In addition to these two requirements, the space environment adds serious complications due to the thermal gradients which are experienced.

Space robots have some requirements that terrestrial robots do not yet have, though we humans do. The capture of what is known as a 'free-flyer' in space, i.e. a spinning satellite is slightly comparable to a human capturing a football or baseball. The space robotic 'free-flyer' problem is probably a bit tougher, because it requires reaction of very large torques $(\sim 600 \mathrm{Nm})$. We do not yet expect terrestrial robots to capture 'free flyers'.
The HMI problem is in providing the force information to the operator in useful real-time format. The robotic space arm tasks are done fairly slowly, but with a force data rate of $10 \mathrm{~Hz}, 6$ degrees of freedom force data is not easy to utilize. Our human system of force sensing is very effective even and complements our visual system. The Human teleoperators (e.g. astronauts) might best be served by force HMI that complements rather than distracts from their visual processing of camera views.

There have been other non-visual HMI alternatives, similar to our human force system, for example, force feedback hand controllers. There are some problems with these systems if one is using the robot for long term tasks involving large force levels - the human teleoperator tires out long before the robot.

The need for force sensing in teleoperation is less driven by capturing free-flyer's than it is by moving large payloads around in somewhat constrained quarters. The Canadarm is 50 feet long, with joint locations pretty similar to our own arm (shoulder, elbow and wrist). It is always possible to see where the arm tip (end effector) is, because there is a camera on the end effector (there is also an elbow camera). But the payload itself may obscure obstacles it may contact (e.g. shuttle cargo bay). The force sensor would provide a means of identifying if an interference has occurred - and a means of backing away from the obstacle without damage or jam.

The Canadarm has functioned well, even without a force sensor, since 1981. There was one experiment, STS-62 [2], where a FMS was added to the Canadarm. The Canadarm2, the 'space station' crane has been built with force sensing capability but it has not been operationally utilized yet. The SPDM, another robot soon to be delivered to International Space Station also has force sensing capability. The force sensing HMI's for these two devices are graphically presented to operators.

\section{Audio Data Presentation}

Human recognition of sound can be broken into three groups: pitch (frequency), intensity (amplitude or loudness) and timbre (complexities of component frequencies). In addition to being able to differentiate sounds on these bases, humans are remarkably good at localizing sound in 3-D, with the exception of an area above and behind our head, the cone of confusion, Figure 2. 
It is this ability to localize sound that sparked our

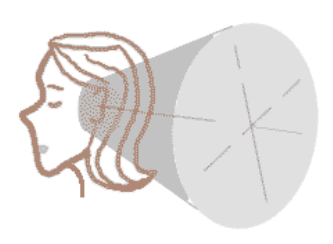

Figure 2

Human audio system - cone of confusion

inspiration into its usefulness as a multi-degree of freedom HMI. Sound has been used as HMI by others in a variety of applications. Flower and associates have employed it as a means of representing time series amplitude information in place of visual, on the basis of musical presentation [4],[5]. Sheridan et al [3] applies a combination of auditory and tactile stimulation to the robotic force feedback problem.

The robotic force feedback problem presents a problem of wide dynamic range requirement (1.5 $\mathrm{Nm}-3000 \mathrm{Nm}$ ). The pitch (frequency) element of music presents a solution to the dynamic range requirement and the timbre (such as distinguishable between musical instruments) allows us to extend the 3-D nature of sound, to cover the 6-D nature required for force. The low level amplitude forces are to be represented as low frequency sound - increasing frequency represents increasing force levels. The distinction between the 3 linear force degrees of freedom and the 3 moment degrees of freedom is met with the use of timbre - i.e. two different musical instruments.

Prior to writing the software to demonstrate the potential of the method, some limited auditory experimental evaluations were performed [1] and [8], to assure ourselves of the suitability of frequency sweep in combination with directionality as a means of intuitive presentation.

The experimental evaluations performed were for a set of 3 humans. We were attempting to establish the 'intuitiveness' of their ability to utilize sounds on the basis of frequency, direction and amplitude. The subjects received no training This was intended to provide the most intuitive environment.

The subjects responses were tested for: 1) frequency sweep over $500-3000 \mathrm{~Hz}$ (ability to detect the magnitude and sense of the sweep range) 2) azimuth/amplitude sensitivity (ability to sense loudness and direction). Amplitude range from 0 to $30 \mathrm{~dB}$.

3) azimuth/frequency sensitivity (ability to sense frequency magnitude and direction). Frequency range from $250-1750 \mathrm{~Hz}$.

Table 1 presents the summarized measured error values for each test.

Table 1

Human Errors for Sound Magnitude and Localization

\begin{tabular}{|l|l|l|}
\hline $\begin{array}{l}\text { Frequency } \\
\text { Sweep }\end{array}$ & $\begin{array}{l}\text { Azimuth \& } \\
\text { Amplitude }\end{array}$ & $\begin{array}{l}\text { Azimuth \& } \\
\text { Frequency }\end{array}$ \\
\hline $15 \%$ & $24 \%$ & $17 \%$ \\
\hline
\end{tabular}

There is no clear indicator that our concept was either viable or optimal. These results were only intended as initial indicators.

The force to audio demonstration software which was developed converts force to sound. The numerical determination of which octave a force is represented by is taken from the formula:

$n=\left[\frac{\log (\text { force })}{\log 2}+1\right]$ where $\mathrm{n}$ is an integer representing octave number. A doubling of force is 1 Octave.

A 2 dimensional software demonstration for the use of audio presentation to a simulated robotic peg-inhole task is shown in Figure 3.

The demo software assumed a linear force range of 0 $450 \mathrm{~N}$ and a moment range of $350 \mathrm{~N}-\mathrm{m}$.

The first view of Figure 3 is the start position of the peg with respect to block with tight fitting hole. The $2^{\text {nd }}$ view shows one linear, one rotation arrow (downward to the left) which represent the magnitude of the contact forces (linear and moment forces). The 3rd view is the peg sliding into the hole - the upward arrow represents the commanded velocity of the peg. There are remaining friction and normal forces as it 
slides in the hole.
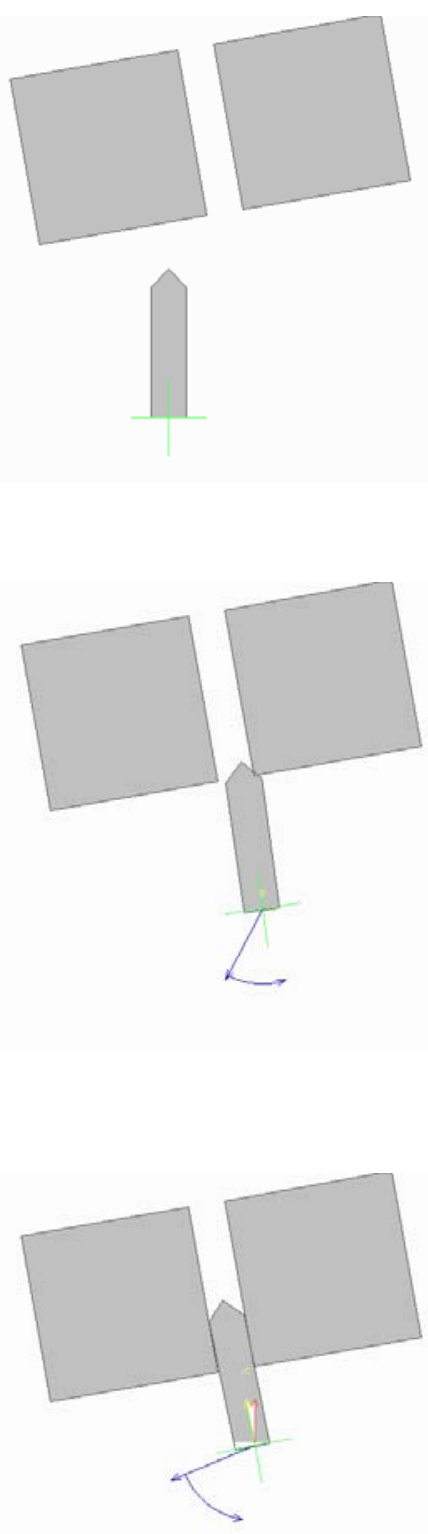

Figure 3

Peg-in-hole task 'screen views'

After successful task completion, a window displays the time taken to complete the task, and the maximum forces generated during the task. The audio signals which are generated are sufficient for an operator to complete the task without visual input - but it takes a bit of practice. The audio data is presented as two musical instruments - one instrument for the linear force direction, the other for the moment degrees of freedom (demonstration software, stearsim, available at www.gve.on.ca). The force magnitude data is presented as high frequency for large forces and low frequency for small forces.

\section{Audio/Vestibular Presentation}

The usefulness of audio presentation of 6 dof force data has some limits when the force directions to be detected are equivalent to somewhere above and behind human head - i.e. cone of confusion, as shown in Figure 2. The cone represents geometries of sound source with theoretically produce identical IID (interaural intensity difference) and ITD (interaural time difference).

The human vestibular system is another system which is very directionally sensitive (it is the sensor system that lets us know if we're leaning or about to tip over). It is possible to artificially stimulate the human vestibular system with surface applied current - i.e. galvanic stimulation, applied behind subjects ears. The mapping of this type of stimulation to humans is an active area of research for psychology researchers (particularly in the visual/vestibular fields such as virtual reality), Inglis et al, [7].

For our robotic HMI application, it offers the ability to improve the directional integrity of audio data presentation.

The audio systems needed for such an HMI application are readily available, but programmable vestibular stimulation is not. To meet this need, we began development of a 'vestibulator'. Its development has been based on a wireless enhancement, to give it a widespread research application.

Prior to our wireless vestibulator development, we participated in some simple vestibulator experiments. A commercial current generator was used to apply current levels between 0.1-1.0 mA to 6 human subjects for stimulus durations between 0.5-3.0 seconds. Current levels above $0.3 \mathrm{~mA}$ produced clear and consistent results in 5 of the 6 subjects. The subjects swayed in the direction of the anode. These results were duplications of work which has been done by Inglis and many others. 


\section{Vestibulator Development}

We have now developed 4 vestibulator prototypes. They are all based on the same PIC (PIC16C63 controller) software, activated and controlled from a PC. The PC host software is available in either Windows or JAVA form.

The first development was a version which could either receive PC generated data via a hardwire link (RS-232 serial link), or by storing script files, downloaded via the hardwire link, for subsequent activation by human subject.

This 'root' system has 5 operating modes, to prevent inappropriate commands from being executed at the wrong time. Table 2 shows the functionality of each mode.

Table 2 Operating Modes

\begin{tabular}{|l|l|l|l|l|}
\hline Mode & $\begin{array}{l}\text { Electrode } \\
\text { Actions }\end{array}$ & $\begin{array}{l}\text { Script } \\
\text { Upload }\end{array}$ & $\begin{array}{l}\text { Script } \\
\text { Running }\end{array}$ & $\begin{array}{l}\text { Tilt } \\
\text { Sensor }\end{array}$ \\
\hline Idle & No & No & No & Yes \\
\hline Direct & Yes & No & No & Yes \\
\hline Program & No & Yes & No & Yes \\
\hline Run & No & No & Yes & No $^{1}$ \\
\hline Fault & No & No & No & No \\
\hline
\end{tabular}

1) Control through the Host software is not allowed but access is available using script commands

The second version is identical to the first, except that a truly real time wireless connection has been implemented using $2 \mathrm{r} / \mathrm{f}$ wireless serial connection devices (Proxim Inc).

The third prototype was developed for potential use on International Space Station (ISS). The target market being researchers interested in isolating the 1$\mathrm{G}$ parameter for mapping studies between electrical stimulation and human response. The ISS version, drawings shown in Figure 4, was modified mechanically by the development of a housing which is stronger and somewhat astronaut friendly (e.g. no sharp corners).

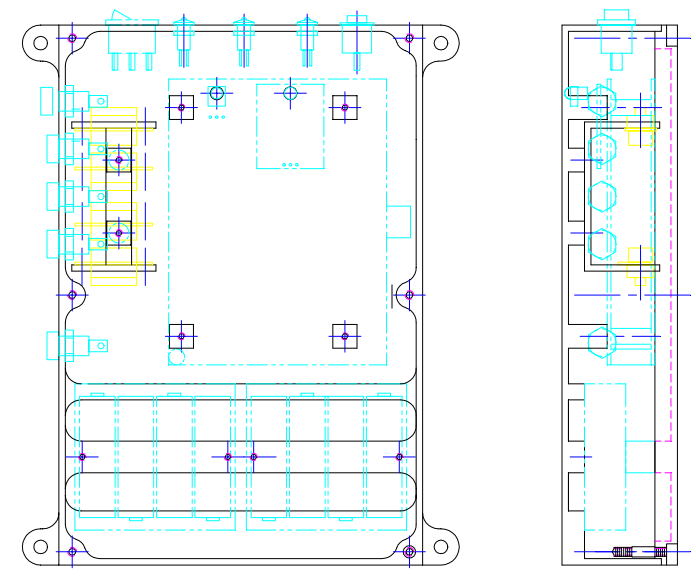

Figure 4 ISS Vestibulator

It is capable of surviving space shuttle launch vibrations, 6.6 grms (Figure 5, vibrations spectra). It was modified electrically by developing an $\mathrm{I} / \mathrm{R}$ wireless link (to reduce potential electrio magnetic interference problems on ISS).

The infrared link was developed with an I/R LED as part of a Fire-Stick II (Rentron) transmitter and a standard infrared detector module as receiver.

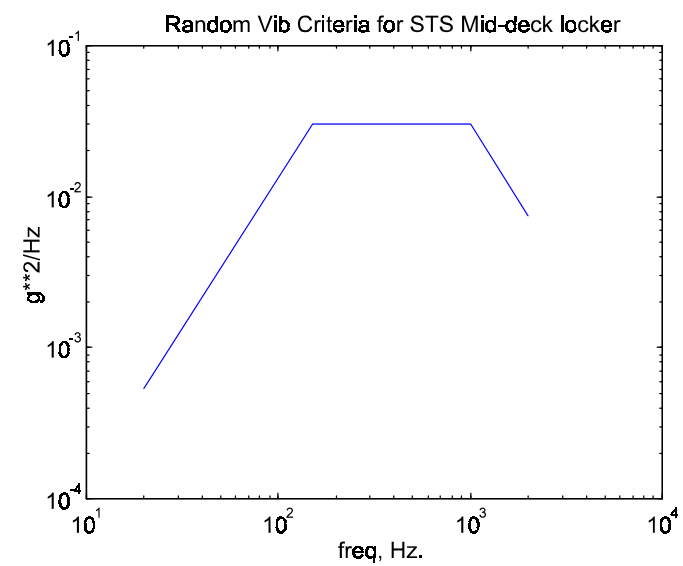

Figure 5 - Vibration Test Spectrum

For the fourth version (based on terrestrial developments) head mounted accelerometers have been added, to allow researchers to monitor head motion, based on applied stimulation. Our York University researchers have chosen to utilize this 
initially as a means of calibrating the signal being applied to human subjects

A MATLAB interface capability was also added to both generate subject stimulation data files and to store input and output data. Table 3 presents the specifications for the tilt-sensor version vestibulator.

Table 3 Vestibulator Specifications

\begin{tabular}{|l|l|}
\hline Weight & 600 grams \\
\hline $\begin{array}{l}\text { Power: } \\
\text { Consumption } \\
\text { Source }\end{array}$ & $\begin{array}{l}40-55 \mathrm{~mA} @ 9.5 \mathrm{~V} \\
8 \mathrm{AA} \text { batteries }\end{array}$ \\
\hline $\begin{array}{l}\text { Electrodes: } \\
\text { No. of Sources } \\
\text { Nominal Current } \\
\text { Measured Current }\end{array}$ & 4 \\
\hline Serial Comm. & -2.56 to $+2.54 \mathrm{~mA}$ \\
Data Rate & \\
\hline Scripts & 9600 baud \\
EEPROM Memory & $2 \mathrm{Kilobytes}$ \\
Frame Rate & $40 \mathrm{~Hz}$. \\
\hline Tilt Sensor & \\
Gravity Range & $-2 \mathrm{~g}$ to $+2 \mathrm{~g}$ \\
Analog Noise & 0.456 degrees pp \\
Quantization Nz. & 0.471 degrees pp \\
Resolution & 0.655 degrees \\
Analog BV & $10 \mathrm{~Hz}$. \\
Acquisition Time & $2-4 \mathrm{~ms}(2 \text { axes })^{3}$ \\
\hline
\end{tabular}

1) probability $95.4 \%$

2) root-sum-square of analog and quantization noise

3) Not including data transfer over serial link

The $\mathrm{r} / \mathrm{f}$ wireless data link module is in addition to that of Table 3. It has a weight of 340 grams $(10 \mathrm{~cm}$ $\mathrm{x} 16.5 \mathrm{~cm} \mathrm{x} 2 \mathrm{~cm})$.

Figure 6 is a picture of the tilt sensor vestibulator, headpiece with tilt sensor on left, main vestibulator box on right.

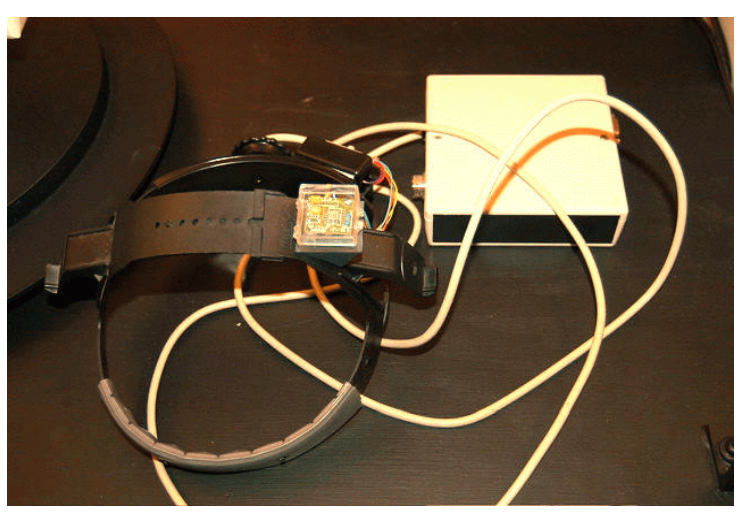

Figure 6

Tilt Sensor Vestibulator

The electrodes and their attachment are something left to the psychology researchers. For testing, we have been using large diameter (1-2") stimulator pads obtained from medical supply stores.

Figure 7 shows the PC host User Interface.

The MATLAB interface is available to generate current vs time text files in a 4 column wide format. It can then be used to generate and upload a script file (time step increments of $25 \mathrm{~ms}$ ).

The tilt sensor must be calibrated between uses. This is done by levelling the system and sending calibrate commands to it, while it is held motionless.

We have been working with Human Performance researchers at York University during the multi-year duration of this project. Their experimental work has been supported by the Ontario Center of Excellence, CRESTECH. The York Researchers have provided us with input to version updates. More importantly though, they perform the human testing which demonstrates the influence of artificial galvanic stimulation on human subjects in their research area (visual/vestibular). Figure 8 is a graph of early results on the type of influence they have measured. Their measurement parameter is based on human subjects ability to distinguish concave and convex shapes in pictures. 


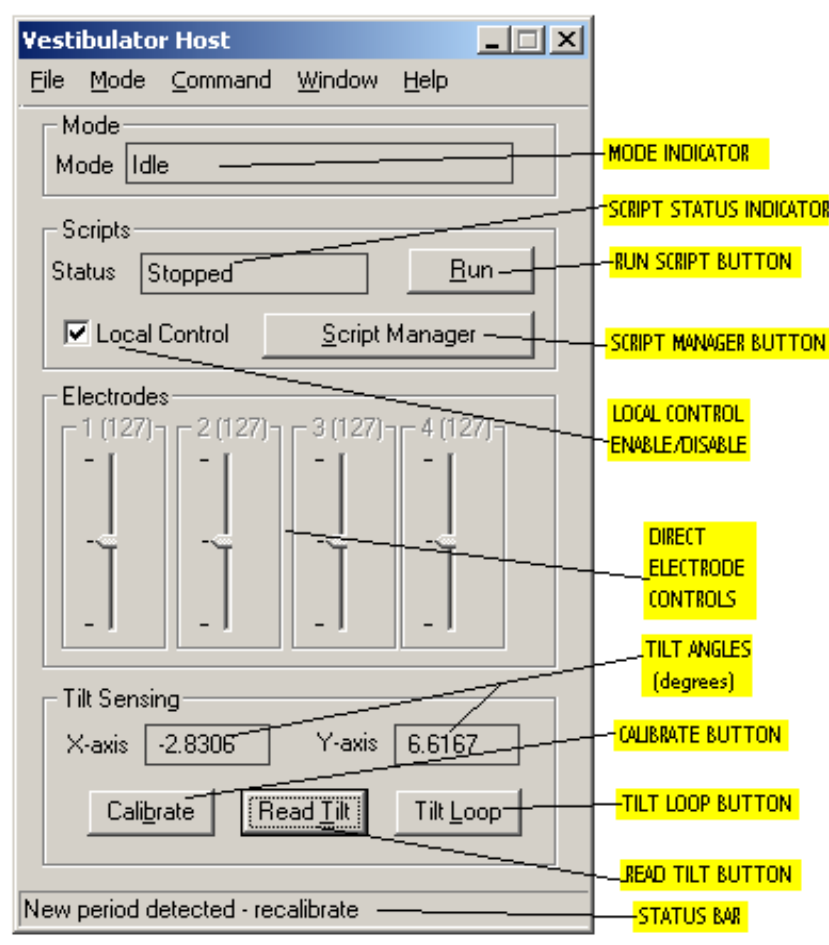

Figure 7

Tilt Sensor User Interface

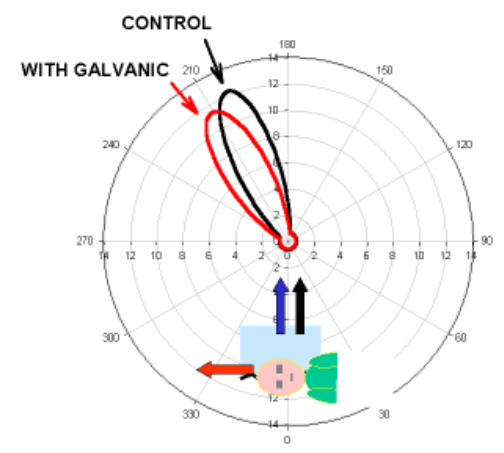

Figure 8

Effect of Vestibulator on Human Perception

\section{Summary}

The design path for this development has not followed standard engineering practice, largely because the problem we were solving was evolving along with the engineering. A new product has been developed and it remains to be seen if it will become widely useful.

In all of our design, we considered the human model and compared it to our requirements. Because we were solving a problem which nature has already solved, that was the obvious path to take. There are two reasons for our diversion from what nature has done. In one case, the robotic capabilities have not progressed to where humans are, in the other case, the robotic requirements are different than we humans have evolved to meet. The lack of gravity is something humans have had no reason to evolve to, as yet, and have not come across any species that has. Unaided humans do not mechanically move large payloads, over long periods of time - a robot is clearly better. But in general, the design assumption was that if nature had already done the optimization then engineering might save a lot of time if it gave natures's designs careful consideration.

\section{References}

[1] Draisey, Sherry. Feasibility Study of MDOFF. Good Vibrations Engineering Report for Stear 10 Phase 1 Study. March

$$
\text { 11, } 1997 .
$$

[2] CDR Data Package, Remote Manipulator System - Force Torque Sensor. NASA JPL, Pasadena CA, 1985.

[3] Massimino and Sheridan (1992). "Using auditory and tactile displays for force feedback." SPIE Vol. 1833 Telemanipulator Technology.

[4] Turnage, Bonebright, Buhman and Flowers (1996). "The effects of Task demands on the equivalence of visual and auditory representations of periodic numerical data”. Behavior Research Methods, Instruments \& Computers.

[5] Flower and Hauer (1995). "Musical vs Visual Graphs: Cross-Modal Equivalence in Perception of Time Series Data". Human Factors 37(3), 553-569. 
[6] Draisey. "Feasibility Study of MDOFF, Volume 1". Good Vibrations Engineering Report for Stear 10 Project. 1997.

[7] Inglis, Shupert, Hvlavacka \& Horak. (1995).

"Effect of Galvanic Vestibular Stimulation on Human

Postural Responses during Support Surface

Translations". Journal of Neurophysiology, Vol 73, No. 2.

[8] Draisey. "Audio Test Report, MDOFF, Phase 1". Good Vibrations Engineering Report for Stear 10

Project. 1997. 